\title{
FLOOD CONTROL RESERVOIR SYSTEM DESIGN USING STOCHASTIC PROGRAMMING
}

\author{
András PRÉKOPA \\ Technical University of Budapest \\ and \\ Computer and Automation Institute of the Hungarian Academy of Sciences \\ H-1111 Budapest, Hungary \\ Tamás SzÁntAi \\ Technical University of Budapest \\ H-1111 Budapest, Hungary \\ Received: April 4, 1977; \\ Revised manuscript received: March 8, 1978
}

\begin{abstract}
Mathematically a natural river system is a rooted directed tree where the orientations of the edges coincide with the directions of the streamflows. Assume that in some of the river valleys it is possible to build reservoirs the purpose of which will be to retain the flood, once a year, say. The problem is to find optimal reservoir capacities by minimizing total building cost eventually plus a penalty, where a reliability type constraint, further lower and upper bounds for the capacities are prescribed. The solution of the obtained nonlinear programming problem is based on the supporting hyperplane method of Veinott combined with simulation of multivariate probability distributions. Numerical illustrations are given.
\end{abstract}

Keywords: Stochastic Programming, Simulation, Hydrological Application.

\section{Formulation of the problem}

The problem we are dealing with has various applications. The most immediate among these is the application for flood control reservoir system design. Hence we shall use terms corresponding to this in the sequel. 
In the theory of graphs the tree is usually defined as a connected undirected graph without circuit. When a special vertex has been designated and called the root of the tree then the tree is a rooted tree.

Taking into account the physical problem we are going to formulate it will be convenient for us to define rooted directed tree which arises from a rooted tree in such a way that a direction is assigned to every edge. The directions we assign are inductively given as follows: starting from the root, select the neighbouring vertices and assign to every edge thus obtained the direction showing in the direction of the root; then we start from the neighbouring vertices and do the same etc. This will be our mathematical model for a natural river system.

Assume that the number of vertices in $n+1$ and denote them by $a_{1}, \ldots, a_{n+1}$. The vertex $a_{i}$ will be called an antecedent of the vertex $a_{j}$ if a directed path leads from $a_{i}$ to $a_{j}$. If a path of this kind exists, then it is clearly unique. If this path consists of $r$ edges, then we say that $a_{i}$ is an antecedent of $a_{j}$ of order $r$. Vertices without antecedents will be called terminal. Edges starting from terminal vertices will also be called terminal. Assume that the terminal vertices are $a_{1}, \ldots, a_{m}$ while $a_{n+1}$ is the root of the tree. We assume further that there is only one edge going into $a_{n+1}$ and this represents such a part of the river system where we can build reservoir. If there would be no possibility here for reservoir building, then we could split up the river system into subsystems and formulate at least two separate flood control problems. Let $a_{n}$ denote that vertex with which the root is connected.

Assume now that we can build reservoirs is some parts of the river system, represented by some edges in our graph model, and the only purpose of the reservoirs will be retaining the flood. This we assume to exist periodically, once a year, say, and to be of a random character. The water comes from terminal points. We assume that the total water quantities can be separated in such a way that we can quantitatively give that amounts which can be lead between river banks and also that amounts which have to be retained by the reservoir system. Throughout the paper we are dealing with the water quantities to be retained only. When retaining the flood, we accept the following policy: first we start to fill up those upstream reservoirs which are located on terminal edges. Then, if the flood cannot be retained by these reservoirs, start to fill up the reservoirs on the next edges by the overflown quantities and those input quantities which arrive on terminal edges without reservoir, etc.

To every terminal vertex there corresponds, a random input water quantity. These will be denoted by $x_{1}, \ldots, x_{m}$. The reservoir capacities to be determined will be denoted by the symbols $K_{i}$. It is convenient to choose the subscript $i$ in such a way that it coincide with the subscript of that vertex from which the edge, having the reservoir with capacity $K_{i}$, starts.

Let us define the quantities $x_{m+1}, \ldots, x_{n+1}$ recursively as follows:

$$
x_{j}=\sum_{i \in A_{j}}\left[x_{i}-\min \left(x_{i}, K_{i}\right)\right]+\sum_{i \in B_{j}} x_{i}, \quad j=m+1, \ldots, n+1,
$$


where $A_{j}\left(B_{j}\right)$ is the collection of those first order antecedents of the vertex $a_{j}$ which are connected with $a_{j}$ by an edge with (without) reservoir.

Now the reservoir system is capable to retain the flood if and only if the following condition holds:

$$
x_{n+1}=0
$$

or what is the same, the inequality holds:

$$
x_{n} \leq K_{n}
$$

Let us introduce the notation

$$
A=\bigcup_{j=m+1}^{n+1} A_{j} .
$$

For the design of the capacities $K_{i}, i \in A$ we formulate the following stochastic programming problem

$$
\begin{aligned}
& \operatorname{minimize} \sum_{i \in A}\left[c_{i}\left(K_{i}\right)+E(\mu)\right] \\
& \text { subject to } \\
& \mathbb{P}\left(x_{n} \leq K_{n}\right) \geq p \\
& 0 \leq K_{i} \leq V_{i}, \quad i \in A
\end{aligned}
$$

where $p$ is a fixed probability, near unity in practice, the $V_{i}, i \in A$ are prescribed numerical upper bounds for the unknown capacities, $E$ is the symbol of expectation, $\mu$ is a random penalty of the wrong deviation $x_{n}-K_{n}>0$ provided it exists and $c_{i}\left(K_{i}\right)$ is the building cost of reservoir $i$. The penalty $\mu$ is defined in the following manner

$$
\mu= \begin{cases}q\left(\kappa_{n}-K_{n}\right), & \text { if } \kappa_{n}>K_{n}, \\ 0, & \text { otherwise }\end{cases}
$$

where $q(z)$ is a nonnegative nondecreasing function on the halfline $z \geq 0$.

Variants of Problem (1.3) may also be of great practical interest. E.g. instead of retaining the flood by the total system of reservoirs we may prescribe the same for a number of subsystems and thus instead of the single inequality $x_{n} \leq K_{n}$ we may have a collection of such inequalities. Then either we prescribe a lower bound for the joint occurrance of these inequalities or use separate probabilistic constraints.

If we impose a positive lower bound $S_{i}$ on $K_{i}$, then by introducing the new variable $L_{i}=K_{i}-S_{i}$, the problem can be reduced to the form (1.3). Instead of $x_{n+1}=0$ we may only require that $x_{n+1} \leq S$ where $S$ is the maximum streamflow which does not cause damage downstream from the root vertex of the directed tree representing the natural hydrological system. This means that instead of $x_{n} \leq K_{n}$ we have to write $x_{n} \leq K_{n}+S$. From here we see that the new problem can be reduced to Problem (1.3) by prescribing the lower bound $S$ for the capacity of reservoir $n$. We have to be careful to write $q(z)=0$ for $0 \leq z \leq S$ in this case. 
The use of the joint probability distribution of the random variables $x_{1}, \ldots, x_{m}$ is fundamental in our reservoir system design methodology. Simple argument concerning simple system shows that the reservoir capacities satisfying a prescribed reliability level strongly depend on the joint probabilistic behaviour of the input water quantities.

\section{Mathematical properties of the reservoir system design model}

First we recall some mathematical concepts and theorems what we need in the sequel.

A nonnegative function $f$ defined on $\mathbb{R}^{k}$ is said to be logarithmic concave (logconcave) if for every $x, y \in \mathbb{R}^{k}$ and $0<\lambda<1$ we have the inequality

$$
f(\lambda x+(1-\lambda) y) \geq[f(x)]^{\lambda}[f(y)]^{1-\lambda} .
$$

If instead of (2.1) we have

$$
f(\lambda x+(1-\lambda) y) \geq \min [f(x), f(y)]
$$

then $f$ is said to be quasi-concave. In the latter case $f$ need not be a nonnegative function. (2.1) obviously implies (2.2).

A probability measure defined on the measurable subsets of the space $\mathbb{R}^{k}$ is said to be logarithmic concave (logconcave) if for every pair $A, B$ of convex subsets of $\mathbb{R}^{k}$ and every $0<\lambda<1$ we have the inequality

$$
P(\lambda A+(1-\lambda) B) \geq[P(A)]^{\lambda}[P(B)]^{1-\lambda} .
$$

If instead of (2.3) we have

$$
P(\lambda A+(1-\lambda) B) \geq \min [P(A), P(B)]
$$

then $P$ is called a quasi-concave probability measure.

Theorem 1. [5, 7]. If the probability measure $P$ is absolutely continuous and is generated by a logconcave probability density function, then $P$ is a logconcave probability measure.

TheOREM 2. [2]. If the probability measure $P$ is absolutely continuous, and is generated by the probability density $f$ for which $f^{-1 / k}$ is a convex function in the entire space $\mathbb{R}^{k}$, then $P$ is a quasi-concave probability measure.

THEOREM 3. [8]. If a random vector y has a logconcave (quasi-concave) probability distribution in $\mathbb{R}^{q}$ and $x=A y+b$, where $A$ is a constant $k \times q$ matrix and $b$ is a constant $k$-component vector, then $x$ has a logconcave (quasi-concave) probability distribution.

The proof is given for the logconcave distribution but the case of a quasiconcave probability distribution needs only trivial modification. 
TheOREm 4. [6]. Let $g_{1}(K, y), \ldots, g_{r}(K, y)$ be concave functions of all variables contained in the vectors $K, y$, where $K \in \mathbb{R}^{k}$ and $y \in \mathbb{R}^{q}$. Assume that $y$ is a random vector having logconcave (quasi-concave) probability distribution. Then

$$
h(K)=\mathbb{P}\left(g_{i}(K, y) \geq 0, i=1, \ldots, r\right)
$$

is a logconcave (quasi-concave) function of the variable $K \in \mathbb{R}^{k}$.

The most common example for logconcave probability distribution is the normal distribution. The nondegenerated normal distribution defined in $\mathbb{R}^{k}$ has the following density function:

$$
f(z)=\left[\frac{1}{(\operatorname{det} C)(2 \pi)^{n}}\right]^{1 / 2} \exp \left\{-\frac{1}{2}(z-t)^{\prime} C^{-1}(z-t)\right\}, \quad z \in \mathbb{R}^{k}
$$

where $C$ is a positive definite matrix, equal to the covariance matrix and $t$ is a real constant vector equal to the expectation vector of the vector valued random variable, $f$ is clearly a logconcave point function hence by Theorem 1 the corresponding probability distribution is logconcave.

A further example will be dealt with in the sequel is a special multivariate gamma distribution introduced in [10]. This is the probability distribution of the random vector $x$ where

$$
x=A y,
$$

the random vector $y$ has independent, standard gamma distributed components and $\mathbf{A}$ is a matrix of 0 and 1 entries. A continuous probability distribution is called standard gamma distribution if it has the following type of probability density:

$$
\frac{1}{\Gamma(\vartheta)} z^{\vartheta-1} e^{-z}, \quad \text { if } \quad z>0
$$

and zero if $z \leq 0 ; \vartheta$ is a positive constant. If $\vartheta \geq 1$, then this density function is logconcave. Assuming this property to hold for the components of $y$ first we realize that $y$ has a logconcave distribution (the independence of the components implies that the joint density is the product of the densities of the components) and by Theorem 3 we derive that $x$ has a logconcave distribution.

If some of the components of $y$ have parameters smaller than 1 , then the probability distribution of $x$ may fail to be logconcave. However, the joint probability distribution function of the components of $x$ is always a logconcave (point) function. We need somewhat more, therefore we prove:

THEOREM 5. If the random vector $y$ has independent, standard gamma distributed components and $A_{1}$ is a matrix with nonnegative entries such that the product $A_{1} y$ can be formed, then the probability distribution function of the random vector $A_{1} y$, i.e. the function

$$
\mathbb{P}\left(A_{1} y \leq z\right)
$$

is logconcave in $z$ in the entire space. 
Proof. Let $y_{i}$ be a component of $y$ and denote by $\vartheta_{i}$ the parameter of its probability distribution. If $\vartheta_{i} \geq 1$, then we leave $y_{i}$ in its original form. If on the other hand $\vartheta_{i}<1$, then we write

$$
y_{i}=\left(y_{i}^{\vartheta_{i}}\right)^{1 / \vartheta_{i}}
$$

and observe that $y_{i}^{\vartheta_{i}}$ has the following probability density function

$$
\begin{aligned}
\frac{\mathrm{d}}{\mathrm{d} z} \mathbb{P}\left(y_{i}^{\vartheta_{i}}<z\right) & =\frac{\mathrm{d}}{\mathrm{d} z} \mathbb{P}\left(y_{i}<z^{1 / \vartheta_{i}}\right) \\
& =\frac{\mathrm{d}}{\mathrm{d} z} \int_{0}^{z^{1 / \vartheta_{i}}} \frac{1}{\Gamma\left(\vartheta_{i}\right)} t^{\vartheta_{i}-1} e^{-t} \mathrm{~d} t \\
& =\frac{1}{\Gamma\left(\vartheta_{i}+1\right)} \exp \left\{-z^{1 / \vartheta_{i}}\right\}
\end{aligned}
$$

for $z>0$ and zero for $z \leq 0$. Since (2.6) is a logconcave probability density and by (2.5) the random variable $y_{i}$ is a convex function of the random variable $y_{i}^{\vartheta_{i}}$ having this logconcave density, Theorem 4 implies that

$$
\mathbb{P}\left(A_{1} y \leq z\right)=\mathbb{P}\left(z-A_{1} y \geq 0\right)
$$

is a logconcave function of the variable $z$. This completes the proof of Theorem 5 .

THEOREM 6. Let y be a random vector having positive valued components and assume that the logarithms of the components have a joint normal distribution. Assume that $A_{1}$ is a matrix with nonnegative entries such that the product $A_{1} y$ can be formed. Then the joint probability distribution of the components of $A_{1} y$ is a logconcave (point) function in the entire space.

Proof. The proof is similar to the proof of Theorem 5. We only have to write

$$
y_{i}=e^{\log y_{i}}
$$

for every component $y_{i}$ of $y$ and repeat the argument applied in the previous proof.

The Gumbel distribution is a favoured distribution to describe the probabilistic behaviour of random extrema. The one-dimensional probability distribution function of a Gumbel distribution has the form

$$
\exp \left\{-\lambda e^{-\mu z}\right\}, \quad-\infty<z<+\infty
$$

where $\lambda>0, \mu>0$ are constants. Taking the derivative we obtain a logconcave (point) function hence this distribution is logconcave.

In our numerical examples the multivariate normal and the multivariate gamma distributions will be used. 
TheOREM 7. Every $x_{j}, j \geq m+1$ is a convex function of those variables among $x_{1}, \ldots, x_{m}$ which belong to vertices antecedent relative to $a_{j}$ and of all $K_{i}$ variables which belong to edges connecting two vertices at least one of which is antecedent relative to $a_{j}$. In particular, if $x$ is the vector of components $x_{1}, \ldots, x_{m}$ and $K$ is the vector of components $K_{i}, i \in A$, then

$$
g(K, x)=K_{n}-x_{n}
$$

is a concave function of $x$ and $K$.

Proof. First we remark that the following equality holds:

$$
x_{i}-\min \left(x_{i}, K_{i}\right)=\max \left(0, x_{i}-K_{i}\right) .
$$

Substituting this into (1.1), a simple induction shows the validity of the theorem. We only have to refer to the fact that the maximum of two convex functions is again a convex function. This proves the theorem.

THEOREM 8. If $x_{1}, \ldots, x_{m}$ have a logconcave (quasi-concave) joint distribution, then the probability

$$
h(K)=\mathbb{P}(g(K, x) \geq 0)
$$

is a logconcave (quasi-concave) function of the variable $K$.

Proof. Theorem 8 is an immediate consequence of Theorem 4 and Theorem 7.

THEOREM 9. If $x$ has a nondegenerated normal distribution, then the function $h(K)$ has continuous gradient at every $K$. If $x$ has the above mentioned multivariate gamma distribution i.e. $x=A y$ where $A$ and $y$ satisfy the mentioned assumptions, then $h(K)$ has continuous gradient except at most for the points where at least one component of $K$ is zero.

PROOF. The condition $x_{n} \leq K_{n}$ can be expressed in terms of linear inequalities so that a number of partial sums of the random variables $x_{1}, \ldots, x_{m}$ are smaller then or equal to some partial sums of the variables $K_{i}, i \in A$. This system of inequalities arises in such a way that we substitute (2.7) into (1.1) and split up subsequently all inequalities of the type $\max (a, b) \leq c$ into the inequalities $a \leq c, b \leq c$. The linear transform of $x$ thus obtained has a matrix which we denote by $B$. This clearly has nonzero rows and every pair of its rows is a pair of linearly independent vectors.

Consider now first the case of the multivariate normal distribution. Since $x$ has a nondegenerated normal distribution, it follows that any two components of $B x$ are linearly independent i.e. the absolute value of their correlation coefficient is smaller than 1 . It is well-known that in this case the joint probability distribution function has continuous gradient in the entire space. Since the probability (2.8) can be expressed so that we put conveniently chosen partial sums of the components of $K$ into the arguments of the joint distribution function of $B x$, our assertion follows. 
In case of the multivariate gamma distribution the argumentation is very similar. Since we cannot guarantee the differentiability of the joint distribution function of $B x$ only at such points where at least one of the components is zero, it follows that if all components of $K$ are positive then the function (2.8) has continuous gradient at the point $K$. This proves the theorem.

THEOREM 10. If the function $q$ is convex, then the penalty term $E(\mu)$ in problem (1.3) is convex no matter what kind of probability distribution $x$ has. If $q$ has continuous derivative and $x$ has a normal joint distribution, then $E(\mu)$ has continuous gradient at every $K$. If $x$ has the multivariate gamma distribution described above, then $E(\mu)$ has a continuous gradient except at most for such $K$ vectors where at least one component is zero, provided $q$ has continuous derivative.

Proof. The theorem can be proved easily on the basis of Theorem (3.1) in [9].

The building costs $c_{i}\left(K_{i}\right)$ are frequently convex in the practice, thus we see that there are conditions, general enough, under which (1.3) is a convex programming problem.

\section{Solution of problem (1.3)}

For the solution of problem (1.3) we use the supporting hyperplane method of VEINOTT [11]. First we summarize this method, then show how it applies to our problem. The following nonlinear programming problem will be considered

$$
\begin{aligned}
& \operatorname{minimize} h_{0}(x), \\
& \text { subject to } \\
& h_{i}(x) \geq 0, \quad i=1, \ldots, s .
\end{aligned}
$$

Assume that the following conditions hold:

Condition 1. There exists a bounded convex polyhedron $K^{1}$ such that

$$
\left\{x \mid h_{i}(x) \geq 0, i=1, \ldots, s\right\} \subset K^{1} .
$$

Condition 2. The functions $-h_{0}, h_{1}, \ldots, h_{s}$ are quasi-concave and have continuous gradient of $K^{1}$.

Condition 3. There exists a $z^{1}$ such that $h_{i}\left(z^{1}\right)>0, i=1, \ldots, s$.

The procedure consists of two phases.

Phase I. Find a vector $z^{1}$ satisfying Condition 3.

Phase II. We perform subsequent iterations where the $r^{\text {th }}$ iteration consists of the following two steps. 
Step 1. Solve the problem

$$
\begin{aligned}
& \text { minimize } h_{0}(x), \\
& \text { subject to } \\
& x \in K^{r}
\end{aligned}
$$

where $K^{r}$ is a bounded convex polyhedron. Let $x^{r}$ be an optimal solution to this problem. If $h_{i}\left(x^{r}\right) \geq 0, i=1, \ldots, s$, then $x^{r}$ is an optimal solution to Problem (3.1). Otherwise go to Step 2.

Step 2. Let $\lambda^{r}$ be the largest $\lambda(0 \leq \lambda \leq 1)$ for which the following inequality holds

$$
h_{i}\left(z^{1}+\lambda\left(x^{r}-z^{1}\right)\right) \geq 0, \quad i=1, \ldots, s .
$$

This one-dimensional problem can be solved e.g. by Fibonacci search. Let

$$
y^{r}=z^{1}+\lambda^{r}\left(x^{r}-z^{1}\right) .
$$

If $h_{0}\left(y^{r}\right)-h_{0}\left(x^{r}\right) \leq \epsilon$, then $y^{r}$ is an approximate solution of Problem (3.1). Otherwise select a subscript for which the equality $h_{i_{r}}\left(y^{r}\right)=0$ holds. Let

$$
K^{r+1}=\left\{x \mid x \in K^{r}, \nabla h_{i_{r}}\left(y^{r}\right)\left(x-y^{r}\right) \geq 0\right\}
$$

and go to Step 1, using $r+1$ instead of $r$.

Figure 1 illustrates one iteration of the above procedure.

VEINOTT has shown that if instead of a fixed vector we use the following sequence

$$
z^{r+1}=z^{r}+\beta\left(y^{r}-z^{r}\right), \quad r=1,2, \ldots
$$

in the subsequent iterations where $0<\beta<1$ is a fixed number, then his method reduces to the modified feasible direction method of Zoutendijk.

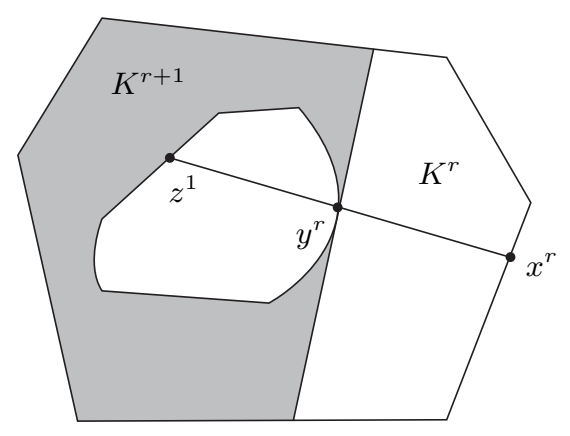

Figure 1: An iterative step in the supporting hyperplane method.

When solving Problem (1.3) we choose

$$
K^{1}=\left\{K \mid 0 \leq K_{i} \leq V_{i}, i \in A\right\} .
$$


We check whether $\mathbb{P}\left(x_{n}<K_{n}\right)>p$ is satisfied in case of $K_{i}=V_{i}, i \in A$. This implies the fulfilment of Condition 3 and the vector of components $K_{i}=V_{i}, i \in A$ is selected to play the role of $z^{1}$. Thus only Phase II has to be performed. The internal point was selected according to relation (3.2) with $\beta=0.5$. Unfortunately the sequence $z^{r}, r=$ $1,2, \ldots$ converged very fast to a boundary point of the set determined by the probabilistic constraint and the procedure stopped before having obtained a good approximation of the optimal solution. Therefore we used the modified relation

$$
z^{r+1}=z^{r}+\frac{1}{r+1}\left(y^{r}-z^{r}\right), \quad r=1,2, \ldots
$$

which improved the method.

The values of the function $\mathbb{P}\left(x_{n} \leq K_{n}\right)$ were obtained by simulation. Since simulation causes numerical inaccuracy, the following cautions were used.

(a) in the course of the one-dimensional optimization we were seeking that $\lambda$ for which the inequality

$$
\mathbb{P}\left(x_{n} \leq K_{n}\right)-p \geq 0.01
$$

holds and the search procedure was stopped in case of an error smaller than 0.005. By this caution the procedure became somewhat slower but we prevented the new cut from cutting down feasible points from the constraining set of Problem (1.3). Primarily the inaccurate evaluation of the gradient causes such a danger. The one-dimensional optimization was a simple interval bisection.

(b) Partial derivatives of the function $\mathbb{P}\left(x_{n} \leq K_{n}\right)$ were computed numerically by the use of simulation corresponding to the values $K_{i}+0.1$ and $K_{i}-0.1$ for every $i \in A$ and the same random numbers were used to compute one gradient value.

The number of samples used for the simulation was 1000 when computing function values and 2000 when computing the derivatives of the probabilistic constraining function.

In case of the multivariate normal distribution the random numbers were generated by the use of a fast algorithm available on the UNIVAC 1108 computer of SCICON Ltd. on which the computations were performed. The method is due to MARSAGLIA and BRAY [3]. The multi-gamma distributed random numbers were generated by the use of algorithm GT of Ahrens and Dieter (see Section 3 in [1]). All programs were written in FORTRAN except for the program generating uniformly distributed random numbers and this latter was written in assembly language.

\section{Numerical examples}

Consider the river system topology given by Figure 2, where the possible reservoir sites are also indicated. The variables defined by (1.1) are the following $\left(x_{1}, x_{2}, x_{3}, x_{4}, x_{5}\right.$ are 


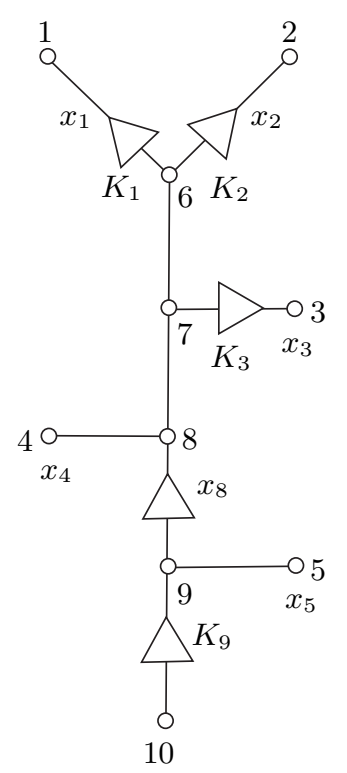

Figure 2: Example for river system topology and possible reservoir sites. Streamflow directions are indicated only on edges without reservoir.

the terminal inputs):

$$
\begin{aligned}
x_{6} & =x_{1}-\min \left(x_{1}, K_{1}\right)+x_{2}-\min \left(x_{2}, K_{2}\right), \\
x_{7} & =x_{3}-\min \left(x_{3}, K_{3}\right)+x_{6}, \\
x_{8} & =x_{4}+x_{7} \\
x_{9} & =x_{8}-\min \left(x_{8}, K_{8}\right)+x_{5}, \\
x_{10} & =x_{9}-\min \left(x_{9}, K_{9}\right) .
\end{aligned}
$$

Thus in terms of terminal variables and capacities, relation (1.2) is the following in this special case

$$
\begin{aligned}
x_{9}= & x_{1}-\min \left(x_{1}, K_{1}\right)+x_{2}-\min \left(x_{2}, K_{2}\right)+x_{3}-\min \left(x_{3}, K_{3}\right)+x_{4} \\
& -\min \left[x_{1}-\min \left(x_{1}, K_{1}\right)+x_{2}-\min \left(x_{2}, K_{2}\right)+x_{3}\right. \\
& \left.-\min \left(x_{3}, K_{3}\right)+x_{4}, K_{8}\right]+x_{5} \leq K_{9} .
\end{aligned}
$$

We shall not use penalty in our examples i.e. choose the penalty function equal to zero. Furthermore, we choose a linear cost function. In practice the cost functions are usually nonlinear. The method described in Section 3 allows the numerical solution of such problems too. Since in this paper we concentrate on the use of joint probabilities in our optimization problem, the above-mentioned simplifications are permissible in the illustrative 
Table 1: Numerical results

\begin{tabular}{cccccccccc}
\hline $\begin{array}{c}\text { Type of } \\
\text { distribution }\end{array}$ & $\begin{array}{c}\text { Correlation } \\
\text { matrix }\end{array}$ & $\begin{array}{c}\text { Probability } \\
\text { level }\end{array}$ & $K_{1}$ & $K_{2}$ & $K_{3}$ & $K_{8}$ & $K_{9}$ & $\begin{array}{c}\text { Objective } \\
\text { function }\end{array}$ & $\begin{array}{c}\text { Computing } \\
\text { time* }\end{array}$ \\
\hline \multirow{3}{*}{$\begin{array}{c}\text { Multivariate } \\
\text { gamma }\end{array}$} & $R_{1}$ & $p=0.8$ & 0.807 & 1 & 1 & 1.356 & 1.412 & 5.591 & $00: 52: 657$ \\
& & $p=0.9$ & 0.751 & 1 & 1 & 1.976 & 1.398 & 6.289 & $00: 35: 688$ \\
& $R_{3}$ & $p=0.8$ & 1 & 1 & 1 & 1.539 & 1.193 & 5.494 & $00: 16: 785$ \\
& & $p=0.9$ & 1 & 1 & 1 & 1.268 & 1.848 & 6.348 & $00: 11: 343$ \\
\hline \multirow{2}{*}{$R_{1}$} & $p=0.8$ & 0.796 & 1 & 1 & 1.591 & 1.383 & 5.816 & $01: 03: 444$ \\
Multivariate & \multirow{2}{*}{$R_{2}$} & $p=0.9$ & 0.998 & 1 & 1 & 1.885 & 1.524 & 6.505 & $00: 25: 126$ \\
& & $p=0.8$ & 0.906 & 1 & 1 & 1.351 & 1.371 & 5.551 & $00: 58: 078$ \\
& \multirow{2}{*}{$R_{3}$} & $p=0.8$ & 1 & 1 & 1 & 1.226 & 1.431 & 5.547 & $00: 51: 426$ \\
& & $p=0.9$ & 1 & 1 & 1 & 1.650 & 1.374 & 5.953 & $00: 57: 461$ \\
\hline
\end{tabular}

${ }^{*}$ Time in minutes/seconds/milliseconds.

examples. Our problem is the following:

$$
\begin{aligned}
& \operatorname{minimize}\left(0.4 K_{1}+0.5 K_{2}+0.6 K_{3}+1.2 K_{8}+1.8 K_{9}\right), \\
& \text { subject to } \\
& \mathbb{P}\left(x_{9} \leq K_{9}\right) \geq p, \quad 0 \leq K_{1} \leq 1, \\
& 0 \leq K_{2} \leq 1, \quad 0 \leq K_{3} \leq 1, \\
& 0 \leq K_{8} \leq 2, \quad 0 \leq K_{9} \leq 3,
\end{aligned}
$$

where $p$ will be chosen 0.8 and 0.9 in the following examples. Altogether 10 numerical examples are presented in Table 1 . The following correlation matrices are used containing correlations of the random variables $x_{1}, x_{2}, x_{3}, x_{4}, x_{5}$ :

$$
\begin{aligned}
& R_{1}=\left(\begin{array}{rrrrr}
1.0 & 0.0 & 0.6 & 0.4 & 0.0 \\
0.0 & 1.0 & 0.5 & 0.3 & 0.3 \\
0.6 & 0.5 & 1.0 & 0.7 & 0.6 \\
0.4 & 0.3 & 0.7 & 1.0 & 0.4 \\
0.0 & 0.3 & 0.6 & 0.4 & 1.0
\end{array}\right), \\
& R_{2}=\left(\begin{array}{rrrrr}
1.0 & -0.5 & 0.0 & 0.3 & -0.5 \\
-0.5 & 1.0 & -0.8 & 0.0 & 0.2 \\
0.0 & -0.8 & 1.0 & 0.0 & 0.3 \\
0.3 & 0.0 & 0.0 & 1.0 & 0.0 \\
-0.5 & 0.2 & 0.3 & 0.0 & 1.0
\end{array}\right), \\
& R_{3}=E
\end{aligned}
$$

where $E$ is the $5 \times 5$ unit matrix. The expectations and standard deviations are the same in all examples. They are (expressed in a certain unit) shown in Table 2. 
Table 2:

\begin{tabular}{ccc}
\hline & Expectations & Standard deviations \\
\hline$x_{1}$ & 0.8 & 0.2 \\
$x_{2}$ & 1.5 & 0.3 \\
$x_{3}$ & 1.2 & 0.6 \\
$x_{4}$ & 0.5 & 0.4 \\
$x_{5}$ & 0.7 & 0.3 \\
\hline
\end{tabular}

For the case of the multigamma distribution we use the representation technique described in [10]. Of course only the case of $R_{1}$ has to be considered because $R_{2}$ contains negative entries too and the case of $R_{3}$ is trivial. For the case of $R_{1}$ we obtain the representation:

$$
\begin{aligned}
& x_{1}=\frac{1}{20}\left(y_{1}+y_{2}+y_{3}\right), \quad x_{2}=\frac{3}{50}\left(y_{4}+y_{5}+y_{6}+y_{7}\right), \\
& x_{3}=\frac{3}{10}\left(y_{1}+y_{4}+y_{5}+y_{8}+y_{9}+y_{10}+y_{11}\right), \\
& x_{4}=\frac{8}{25}\left(y_{2}+y_{6}+y_{8}+y_{9}+y_{12}\right), \\
& x_{5}=\frac{9}{70}\left(y_{4}+y_{8}+y_{10}+y_{13}\right),
\end{aligned}
$$

where $y_{1}, \ldots, y_{13}$ have standard gamma distributions with the following parameters:

$$
\begin{array}{lll}
\vartheta_{1}=0.576 & \vartheta_{6}=0.225 & \vartheta_{10}=0.050 \\
\vartheta_{2}=0.160 & \vartheta_{7}=23.875 & \vartheta_{11}=2.055 \\
\vartheta_{3}=15.264 & \vartheta_{8}=0.140 & \vartheta_{12}=0.758 \\
\vartheta_{4}=0.315 & \vartheta_{9}=0.280 & \vartheta_{13}=4.940 \\
\vartheta_{5}=0.585 & &
\end{array}
$$

For the sake of completeness we give the system of inequalities containing partial sums of $x_{1}, x_{2}, x_{3}, x_{4}, x_{5}$ and $K_{1}, K_{2}, K_{3}, K_{8}, K_{9}$ which are together equivalent to the single inequality $x_{9} \leq K_{9}$ :

$$
\begin{aligned}
& x_{5} \leq \quad K_{9}, \\
& x_{4}+x_{5} \leq \quad K_{8}+K_{9}, \\
& x_{1}+x_{4}+x_{5} \leq K_{1}+K_{8}+K_{9}, \\
& x_{2}+x_{4}+x_{5} \leq \quad K_{2} \quad+K_{8}+K_{9}, \\
& x_{3}+x_{4}+x_{5} \leq \quad K_{3}+K_{8}+K_{9}, \\
& x_{1}+x_{2}+x_{4}+x_{5} \leq K_{1}+K_{2}+K_{8}+K_{9}, \\
& x_{1}+x_{3}+x_{4}+x_{5} \leq K_{1} \quad+K_{3}+K_{8}+K_{9}, \\
& x_{2}+x_{3}+x_{4}+x_{5} \leq \quad K_{2}+K_{3}+K_{8}+K_{9} \text {, } \\
& x_{1}+x_{2}+x_{3}+x_{4}+x_{5} \leq K_{1}+K_{2}+K_{3}+K_{8}+K_{9} \text {. }
\end{aligned}
$$


The optimal solutions, optimal objective function values and computing times are contained in Table 1.

\section{Acknowledgement}

The authors express their thanks to Martin Beale who kindly offered the use of the UNIVAC 1108 computer of SCICON Ltd.

\section{References}

[1] Ahrens, J. H. and U. Dieter (1974). Computer methods for sampling from gamma, beta, Poisson and binomial distributions. Computing, 12, 223-246.

[2] Borell, C. (1975). Convex set functions in $d$-space. Periodica Mathematica Hungarica, 6, 111-136.

[3] Marsaglia, G. and T. A. Bray (1964). A convenient method for generating normal variables. SIAM Review, 6, 260-264.

[4] Ore, O. (1962). Theory of graphs. American Mathematical Society Coll. Publ., 38.

[5] PrÉkopa, A. (1971). Logarithmic concave measures with application to stochastic programming. Acta Scientiarum Mathematicarum, 32, 301-316.

[6] PrÉkopa, A. (1972). A class of stochastic programming decision problems. Mathematische Operations-forschung und Statistik, 3, 349-354.

[7] Prékopa, A. On logarithmic concave measures and functions. Acta Scientiarum Mathematicarum, 34, 335-343.

[8] Prékopa, A. (1973). Stochastic programming models for inventory control and water storage problems. In: Inventory control and water storage, Coll. Math. Soc. J. Bolyai 7 (North-Holland, Amsterdam), 229-245.

[9] Prékopa, A. (1973). Contributions to the theory of stochastic programming. Mathematical Programming, 4, 202-221.

[10] PrÉkopa, A. and T. SzÁntai (1978). A new multivariate gamma distribution and its fitting to empirical data. Water Resources Research, 14, 19-24.

[11] Veinoti, A. F. (1967). The supporting hyperplane method for unimodal programming. Operations Research, 147-152. 